\title{
Resveratrol suppresses the STAT3 signaling pathway and inhibits proliferation of high glucose-exposed HepG2 cells partly through SIRT1
}

\author{
YONGGUANG LI, WEI ZHU, JINGBO LI, MINGYA LIU and MENG WEI \\ Department of Cardiology, Sixth Hospital, Shanghai Jiao Tong University, \\ State Key Discipline of Cardiology, Shanghai 200233, P.R. China
}

Received July 1, 2013; Accepted August 21, 2013

DOI: $10.3892 /$ or.2013.2748

\begin{abstract}
Hepatocellular carcinoma is the most common type of liver cancer. The risk of hepatocellular carcinoma for type 2 diabetic patients is greater than that for non-diabetic individuals although the mechanism is unclear. The cancer suppressor resveratrol inhibits cancer cell proliferation partly through the STAT3 signaling pathway. However, the effects of resveratrol on STAT3 in high glucose-exposed HepG2 cells and the role of SIRT1 are not clear to date. The aim of the present study was to investigate the effects of resveratrol on STAT3 and SIRT1 regarding the proliferation of high glucose-exposed HepG 2 cells. HepG 2 cells were cultured in DMEM containing glucose $(2.8,5.5$ and $25 \mathrm{mM})$ and resveratrol $(0,10$ and $100 \mu \mathrm{M})$. HepG 2 cell proliferation and viability were analyzed by MTT assays. The levels of p-STAT3 and SIRT1 were analyzed by western blotting, and RT-PCR methods were used to detect the transcription levels of cyclin B1, cyclin D1, VEGF and MMP-9. SIRT1-specific short-interfering RNA was used to investigate the role of SIRT1 in p-STAT3 signaling. A high glucose concentration $(25 \mathrm{mM})$ induced HepG 2 cell proliferation. This effect was suppressed by resveratrol $(100 \mu \mathrm{M})$, and the effect on the p-STAT3 signaling pathway was found to be SIRT1-dependent. Our findings may provide new insights into the mechanism by which resveratrol suppresses HepG2 cell proliferation under conditions of high glucose. Furthermore, this information may provide the basis for a novel therapeutic strategy for hepatocellular carcinoma patients suffering from either diabetes or hyperglycemia.
\end{abstract}

Correspondence to: Dr Meng Wei, Department of Cardiology, Sixth Hospital, Shanghai Jiao Tong University, State Key Discipline of Cardiology, No. 600 Yishan Road, Shanghai 200233, P.R. China E-mail: weimeng_sjtu6h@163.com

Keywords: resveratrol, hyperglycemia, hepatocellular carcinoma, proliferation, STAT3, SIRT1

\section{Introduction}

Hepatocellular carcinoma (HCC, also called malignant hepatoma) is the most common among the various forms of liver cancer. This tumor also has a variant type that consists of both HCC and cholangiocarcinoma components. The risk of hepatocellular carcinoma in type 2 diabetics is increased by 2.5 to 7.1 times (1) that of the non-diabetic risk depending on the duration of diabetes and treatment protocol, and despite the efforts of scientists and clinicians, the mechanism is not clear.

The novel cancer suppressor, resveratrol is a stilbenoid, which is a type of natural phenol and phytoalexin produced naturally by several plants. Resveratrol is currently the topic of numerous animal and human studies, and although its anticancer effects have been reported, the underlying mechanism remains to be elucidated (2). During recent research, we found that resveratrol inhibits multiple myeloma cell proliferation and induces apoptosis through downregulation of STAT3 (3), which is a member of the STAT signaling protein family (4). In response to cytokines and growth factors, STAT family members are phosphorylated by receptor-associated kinases and then form homodimers or heterodimers that translocate to the cell nucleus, where they act as transcription activators. STAT3 is activated through phosphorylation of tyrosine 705 in response to various cytokines and growth factors including interferons, epidermal growth factor, interleukin (IL)-5, IL-6, hepatocyte growth factor, leukemia inhibitory factor (LIF), bone morphogenetic protein 2 and also the hormone leptin. STAT3 regulates the expression of a variety of genes in response to cell stimuli, and thus plays a key role in many cellular processes such as cell growth and apoptosis (5). The downregulation of STAT3 in resveratrol-treated medulloblastoma cells confirmed the growth inhibition effects of this drug (6).

Resveratrol can induce the expression of SIRT1, which is a longevity protein. SIRT1 can inhibit the proliferation of pancreatic cancer cells (7), and the downregulation of SIRT1 in hAT MSCs induces senescence and inhibits cell proliferation (8). STAT3 phosphorylation and function in the liver were found to be tightly regulated by the nutritional status of an animal, through SIRT1-mediated deacetylation of key STAT3 
Table I Oligonucleotides used in the PCR assay.

Name

Sequence $\left(5^{\prime}-3^{\prime}\right)$

Product (bp)

homo_MMP9_F
homo_MMP9_R
homo_actin_F
homo_actin_R
homo_cyclin B1_F
homo_cyclin B1_R
homo_cycllin D1_F
homo_cycllin D1_R
home_VEGF_F1
home_VEGF_R1

ACACCGACGACCGGTTTGGC
TCGAGTCAGCTCGGGTCGGG
AGCCTCGCCTTTGCCGATCC
ACATGCCGGAGCCGTTGTCG
GCAGCACCTGGCTAAGAATGT
GCCTTGGCTAAATCTTGAACT
GCGAGGAACAGAAGTGCG
AGGCGGTAGTAGGACAGGAA
ACGGACAGACAGACAGACACC
CCCAGAAGTTGGACGAAAAGT

F, forward; R, reverse.

lysine sites (9). Yet, the relationship among resveratrol, SIRT1 and STAT3 in HepG2 cells is not clear.

In the present study, we found that resveratrol suppressed proliferation and cell viability of high glucose-exposed HepG2 cells. STAT3 was constitutively expressed in human HepG2 cells under a high glucose condition; therefore, we investigated the effects of resveratrol on the proliferation and invasive activity of HepG2 cells. The results showed that high glucose induced increased expression of STAT3 and that resveratrol suppressed the proliferation and invasive activity of high glucose-exposed HepG2 cells. Furthermore, resveratrol suppressed the expression of MMP-9, VEGF and cyclin B1, but not cyclin D1. These data demonstrate that resveratrol suppresses the proliferation of high glucose-exposed HepG2 cells and indicate that this effect is mediated via the STAT3 signaling pathway. The present study also demonstrated that the effect of resveratrol on the STAT3 and AKT signaling pathways was SIRT1-dependent.

\section{Materials and methods}

Materials. Resveratrol (0.1\% DMSO) was purchased from Sigma (St. Louis, MO, USA), and the MTT cell proliferation and cytotoxicity assay kit was purchased from Sangon Biotech (Shanghai, China). The Matrigel invasion chamber was purchased from Becton-Dickinson Labware (Bedford, MA, USA). Antibodies against STAT3 and STAT3 phosphorylated at tyrosine 705 and ser727, AKT and AKT phosphorylated at tyrosine 473, SIRT1 and the SIRT1 suppressor EX527 were purchased from Cell Signaling Technology (Danvers, MA, USA); secondary antibodies were purchased from Beijing Biosynthesis Biotechnology (Beijing, China). Penicillin, streptomycin, DMEM, and fetal bovine serum (FBS) were obtained from Gibco (Grand Island, NY, USA).

Cell culture. HepG2 cells, which belong to the classic subclass of human HCC, were obtained from the American Type Culture Collection (ATCC, Manassas, VA, USA). The HepG2 cells were maintained in DMEM containing glucose $(2.8,5.5$ and $25 \mathrm{mM}$ ) supplemented with $10 \%$ FBS, L-glutamine and antibiotics (100 U/ml penicillin and $100 \mu \mathrm{g} / \mathrm{ml}$ streptomycin) at $37^{\circ} \mathrm{C}$ in the presence of $5 \% \mathrm{CO}_{2}$. The effect of resveratrol and the role of SIRT1 in HepG2 cells cultured in high glucose DMEM were investigated by the addition of resveratrol $(100 \mu \mathrm{M})$ and EX527 $(40 \mu \mathrm{M})$.

Western blot analysis. Whole cell extracts of resveratroltreated cells were examined for expression of phospho-STAT3, STAT3, phospho-AKT, AKT, SIRT1 and $\beta$-actin by western blot analysis as previously described (3). The whole cell extracts were prepared by lysing resveratrol-treated cells in lysis buffer [20 mM Tris ( $\mathrm{pH} 7.4), 250 \mathrm{mM} \mathrm{NaCl}$, 2 mM EDTA (pH 8.0), 0.1\% Triton X-100, $0.01 \mathrm{mg} / \mathrm{ml}$ aprotinin, $0.005 \mathrm{mg} / \mathrm{ml}$ leupeptin, $0.4 \mathrm{mM}$ phenylmethylsulphonylfluoride and $4 \mathrm{mM}$ sodium orthovanadate). The protein concentration was quantified using BSA protein assay kits. Equal amounts of protein $(40 \mu \mathrm{g})$ were separated by $8-10 \%$ Tris-glycine gel (Novex, Invitrogen, USA) electrophoresis. The proteins were then electro-transferred to a nitrocellulose membrane (Novex), blocked with 5\% non-fat milk and probed with various primary antibodies overnight at $4^{\circ} \mathrm{C}$. The blots were then washed three times ( 5 min per wash), and then exposed to horseradish peroxidase-conjugated secondary antibodies for $2 \mathrm{~h}$. Immunoreactivity was finally examined by an enhanced chemiluminescence (ECL) reagent (Thermo Fisher Scientific, Waltham, MA, USA). The band for each protein was then quantified by densitometry using ImageJ software (version 1.41; NIH, Bethesda, MD, USA) and normalized to the expression of $\beta$-actin for protein loading.

Reverse transcription-PCR analysis. Total RNA was isolated from HepG 2 cells after $24 \mathrm{~h}$ of treatment with resveratrol using TRIzol ${ }^{\circledR}$ reagent (Life Technologies, Rockville, MD, USA) following the manufacturer's protocol. cDNA was synthesized from $1 \mu \mathrm{g}$ of total RNA using a cDNA synthesis kit (Takara Biotechnology Co., Ltd., Dalian, China) following the manufacturer's instructions. Primer sequences (Genencor, Shanghai, China) specific for MMP-9, STAT3, cyclin B1, cyclin D1 and VEGF are shown in Table I. $\beta$-actin was used to normalize the cDNA input levels. After cDNA synthesis, the 

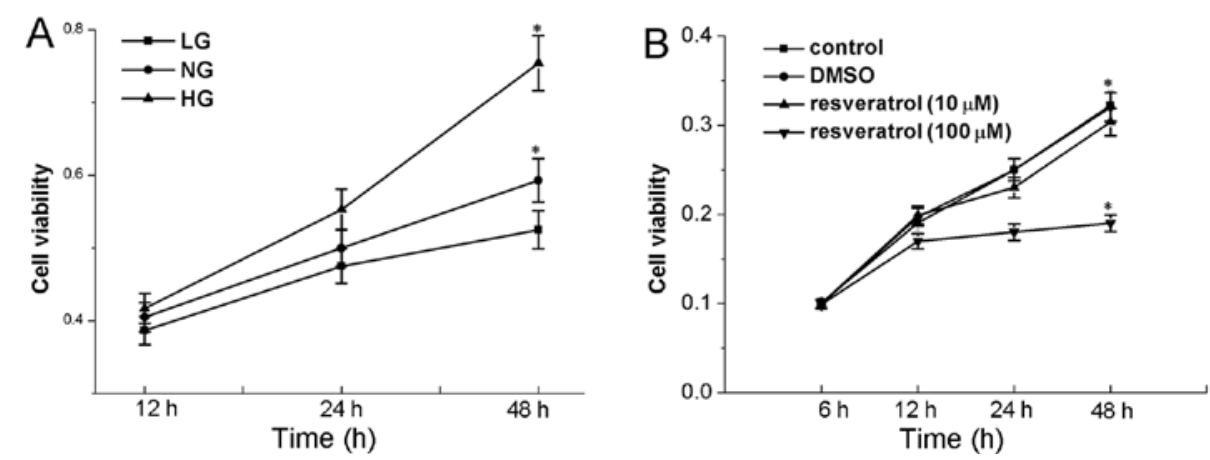

Figure 1. (A) Effect of high glucose on the cell viability of HepG2 cells. HepG2 cells were pretreated with DMEM containing glucose [2.8 (LG), 5.5 (NG) and $25 \mathrm{mM}(\mathrm{HG})]$. After incubation for 12,24 and $48 \mathrm{~h}$ at $37^{\circ} \mathrm{C}$, cell viability was determined by the MTT reduction assay as described in Materials and methods. (B) Effect of resveratrol on cell viability in high glucose-exposed HepG2 cells. After pretreatment with high glucose DMEM (25 mM) for $12 \mathrm{~h}$, and then resveratrol (10 and $100 \mu \mathrm{M}, 0.1 \% \mathrm{DMSO}$ ) for $6,12,24$ and $48 \mathrm{~h}$ at $37^{\circ} \mathrm{C}$, cell viability was determined by the MTT reduction assay. Data are the mean $\pm \mathrm{SD}$ values for three independent experiments ("P<0.05 compared with the control). LG, low glucose; NG, normal glucose; HG, high glucose.

PCR reaction consisted of 32 cycles of denaturation at $95^{\circ} \mathrm{C}$ for $30 \mathrm{sec}$, annealing at $56^{\circ} \mathrm{C}$ for $30 \mathrm{sec}$, extension at $72^{\circ} \mathrm{C}$ for $30 \mathrm{sec}$, and a further $5 \mathrm{~min}$ at $72^{\circ} \mathrm{C}$ in the last cycle. PCR products were separated by electrophoresis on a $2 \%$ agarose gel and visualized by staining with ethidium bromide. Each sample was analyzed in three biological replicates, and at least three reactions were used to calculate the expression. The relative expression was quantified densitometrically using the Gel Image Ver. 3.74 System (Tianon, Shanghai, China).

Migration assay in vitro. Cell migration assays were performed in a Matrigel chamber (Becton-Dickinson Labware). Briefly, $5 \times 10^{4}$ cells in $800 \mu \mathrm{l}$ of serum-free culture medium were added to the upper chamber, while medium supplemented with $10 \%$ FBS and resveratrol was added to the lower chamber. After incubation for $24 \mathrm{~h}$, the cells remaining on the upper side of the filters were removed with a cotton swab. The cells that had migrated to the bottom surface of the filter were fixed with $4 \%$ paraformaldehyde, and stained with $\left(0.1 \% \mathrm{H}_{2} \mathrm{O}\right)$ crystal violet. Stained cells were visualized using an Olympus IX71 microscope (Olympus, Tokyo, Japan).

Transient RNA interference and transfections. SIRT1 was knocked down by using small siRNAs, with a non-targeting siRNA used in parallel as a negative control (GenePharma Co., Shanghai, China). Primary cultures were transfected with siRNA SIRT1 or irrelevant siRNA using X-tremeGENE HP DNA transfection reagent (Roche Diagnostics GmbH/Roche Applied Science, Mannheim, Germany). After 2 days of mRNA silencing, HepG2 cells were analyzed for protein expression of SIRT1, phospho-STAT3, STAT3, phospho-AKT, AKT and $\beta$-actin.

MTT assays in vitro. The antiproliferative effects of resveratrol on HepG2 cells under a high glucose condition were determined by the nicotinamide, 3-(4,5-dimethylthiazol-2-yl)2,5-diphenyltetrazolium bromide (MTT) dye uptake method as previously described (10). Briefly, HepG2 were counted using a hemocytometer and equally distributed in 96 -well plates at a density of $1 \times 10^{4}$ cells/well. Cells were treated with resveratrol at the indicated concentrations $(0,10$ and $100 \mu \mathrm{M})$ for $6,12,24$ and $48 \mathrm{~h}$. To determine cell viability, the DMEM (serum 10\%) was removed, and cells were incubated with MTT (Sigma) at a final concentration of $5 \mathrm{mg} / \mathrm{ml}$ in DMEM for $6 \mathrm{~h}$ in the dark at $37^{\circ} \mathrm{C}$. The supernatant was then removed from each well. The colored formazan crystals produced from MTT were dissolved in $150 \mu 1 \mathrm{DMSO}$, and cell viability was determined spectrophotometrically at $490 \mathrm{~nm}$ (BioTek, Winooski, VT, USA).

Statistical analysis. All results are expressed as means \pm SD relative to the control value. Experiments were performed on three independent occasions. Statistical comparisons between groups were analyzed by t-test or ANOVA followed by Tukey's post-hoc test as appropriate. $\mathrm{P}<0.05$ was considered to indicate a statistically significant result. All data analysis was performed with the use of SPSS 13.0 statistical software.

\section{Results}

Resveratrol reduces the cell viability of high glucose-exposed HepG2 cells. In the present study, we showed that a high glucose concentration $(25 \mathrm{mM})$ increased the proliferation of HepG2 cells when compared to that of the control group $(5.5 \mathrm{mM})$ (Fig. 1A). Furthermore, under a high glucose condition, resveratrol reduced the viability of HepG2 cells at a dose of $100 \mu \mathrm{M}$ but not at $10 \mu \mathrm{M}$ (Fig. 1B).

Resveratrol suppresses the proliferation and invasive capacity of Hep 2 cells. In order to elucidate the relationship between resveratrol and STAT3 in HepG2 cells under a high glucose condition, we analyzed the effect of resveratrol on the proliferation and invasive capacity of high glucose-exposed HepG2 cells. Resveratrol (100 and $200 \mu \mathrm{M})$ suppressed the proliferation of high glucose-exposed HepG2 cells in a dosedependent manner (Fig. 2A and B). Compared with the control DMSO group, resveratrol (100 and $200 \mu \mathrm{M})$ also suppressed the invasive capacity of the HepG2 cells under a high glucose condition (Fig. 2C and D).

Resveratrol suppresses the expression of p-STAT3 in high glucose-exposed HepG2 cells. STAT3 can promote oncogenesis by being constitutively active in various signaling pathways $(11,12)$. Furthermore, STAT3 suppression inhibits human HepG2 cell proliferation in vitro (13). In the present 

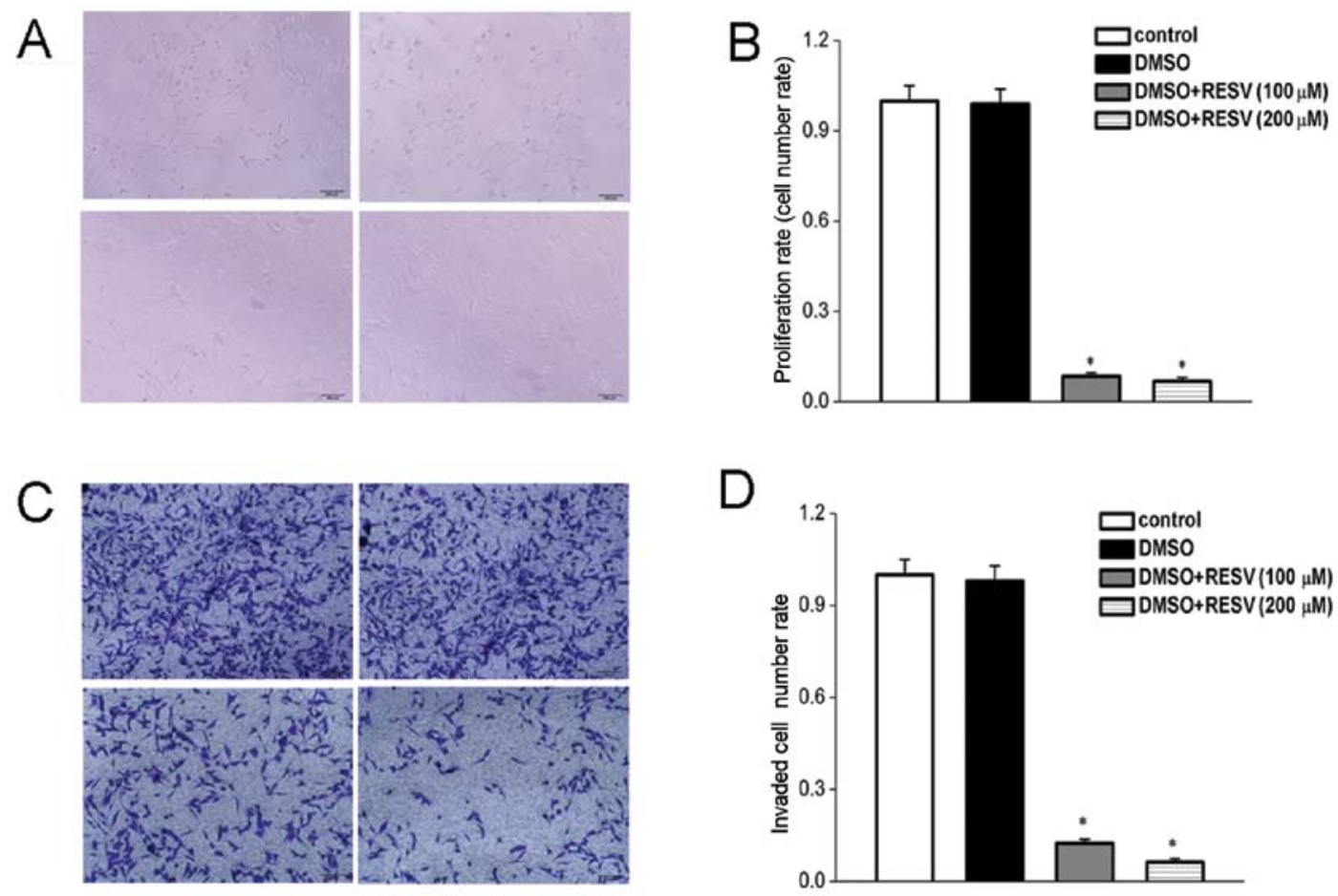

Figure 2. Effect of resveratrol on high glucose-exposed HepG2 cell proliferation and invasion. (A) Resveratrol (100 and $200 \mu \mathrm{M})(0.1 \%$ DMSO) significantly suppressed the proliferation of HepG2 cells after a 48-h incubation. (B) HepG2 cell proliferation was decreased in comparison with the control. Resveratrol $(100$ and $200 \mu \mathrm{M})$ attenuated impairment of high glucose-exposed HepG2 cell proliferation during incubation for $48 \mathrm{~h}$. (C) Cell invasion assays were performed in a Matrigel invasion chamber. After pretreatment of resveratrol for $24 \mathrm{~h}$, cell invasive ability was quantified. Resveratrol (100 and $200 \mu \mathrm{M})$ significantly suppressed the HepG2 cell invasion. (D) HepG2 cell invasion was decreased in comparison with the control. Resveratrol (100 and $200 \mu \mathrm{M})$ significantly suppressed HepG2 cell invasion. Data are the mean $\pm \mathrm{SD}$ values for three independent experiments (" $\mathrm{P}<0.05$ compared with the control).
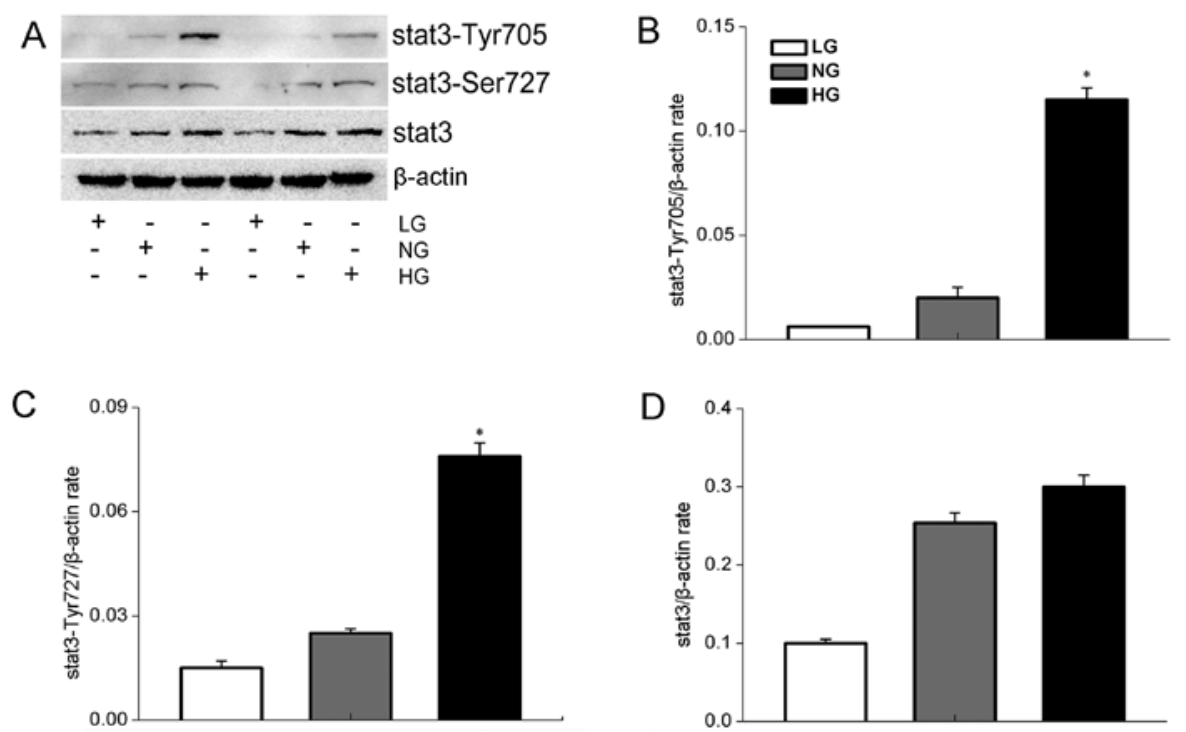

Figure 3. p-STAT3 protein expression in HepG2 cells under different glucose-DMEM conditions. Cells were incubated in normal medium (NG; control) or in low (LG) $(2.8 \mathrm{mM})$ and high glucose (HG) $(25 \mathrm{mM})$ DMEM medium for $12 \mathrm{~h}$. (A) Representative western blots showing p-STAT3, STAT3 and $\beta$-actin in the samples. The bands were quantified, and the results are expressed as the percentage of the control for each protein. (B) STAT3-Tyr705 level; (C) STAT3-Ser727 level and (D) STAT3 level. The results represent the mean \pm SD values for three independent experiments (" $\mathrm{P}<0.05$ compared with the control).

study, we showed that expression of STAT3 and phosphoSTAT3 was induced in high glucose-exposed HepG2 cells, with expression of the phosphorylated form increasing with glucose levels. Thus, phospho-STAT3 expression was induced by a high glucose condition (Fig. 3A).
Investigation of the effect of resveratrol on the STAT3 signaling pathway revealed that resveratrol downregulated the expression of p-STAT3-Tyr705 in a dose-dependent manner, but had no effect on the total level of STAT3 in HepG2 cells (Fig. 4A). 

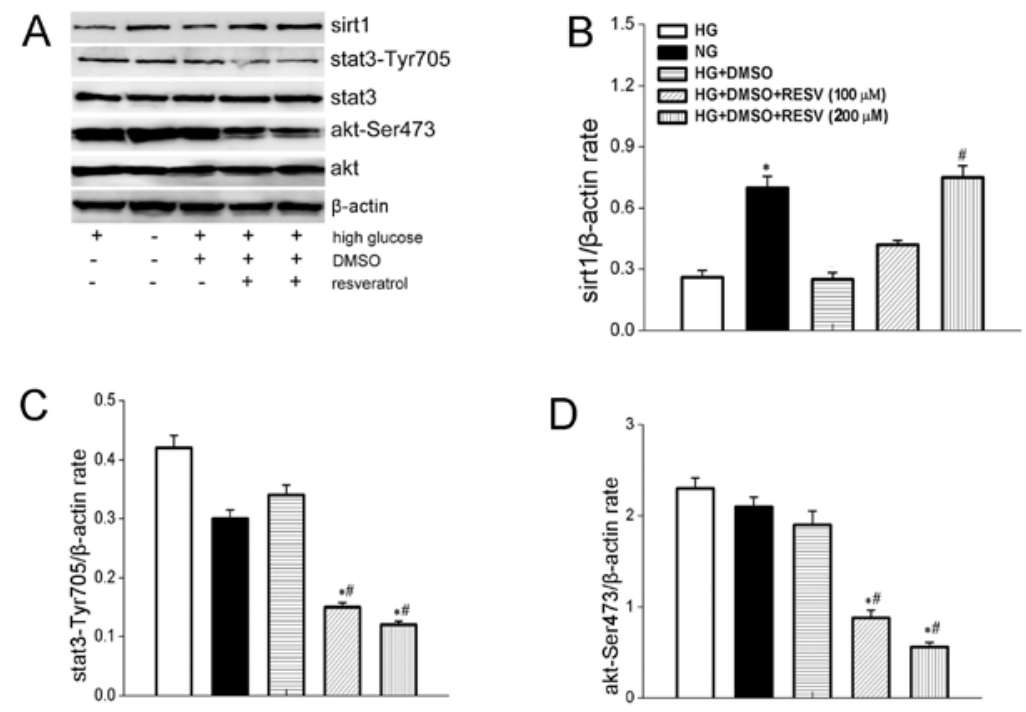

Figure 4. Analysis of the expression of STAT3, p-STAT3, AKT, p-AKT, SIRT1 and $\beta$-actin in high glucose-exposed HepG2 cells treated with resveratrol for $12 \mathrm{~h}$. (B) The abundance of SIRT1 protein is shown as a ratio to $\beta$-actin; (C) the p-STAT3-Tyr705 level is shown as a ratio to $\beta$-actin and (D) the p-AKT-Ser473 level is show as a ratio to $\beta$-actin. The results also represent the mean \pm SD values for three independent experiments ("P $<0.05$ compared with the control; ${ }^{\#} \mathrm{P}<0.05$ compared with the resveratrol groups). HG, high glucose.
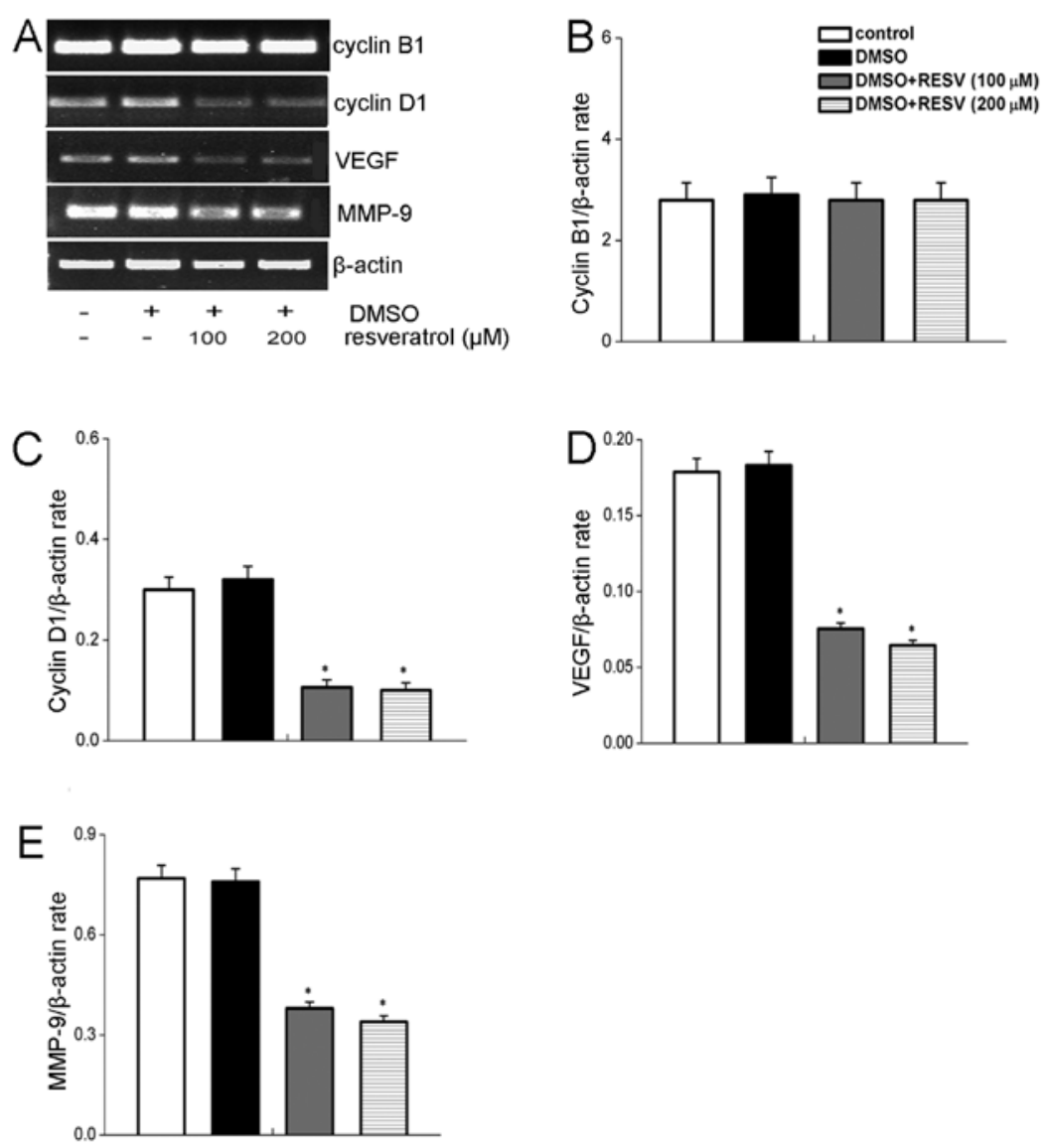

Figure 5. Analysis of the expression of cyclin D1, cyclin B1, VEGF, MMP-9, the downstream genes of STAT3 by RT-PCR. High glucose-exposed HepG2 cells were incubated in high glucose medium (control) or in the presence of resveratrol (100 and $200 \mu \mathrm{M})$ for $12 \mathrm{~h}$. (A) Representative ethidium bromide stained gels showing the target genes and $\beta$-actin in the same sample. (B) The expression of cyclin B1 mRNA is shown as a ratio to $\beta$-actin. (C) The downregulation of cyclin D1 mRNA is shown as a ratio to $\beta$-actin. (D) The downregulation of VEGF mRNA is shown as a ratio to $\beta$-actin. (E) The downregulation of MMP-9 mRNA is shown as a ratio to $\beta$-actin. The results represent the mean \pm SD values for three independent determinations (" $\mathrm{P}<0.05$ compared with the control). 
A

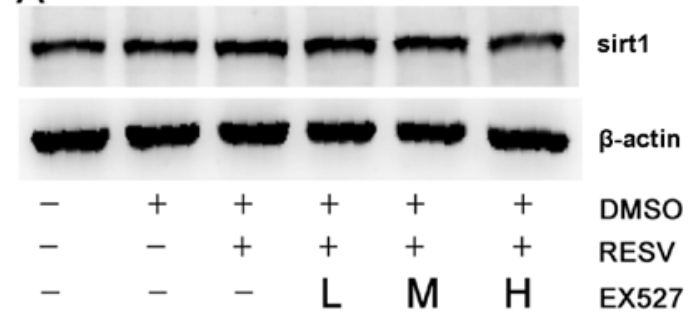

B

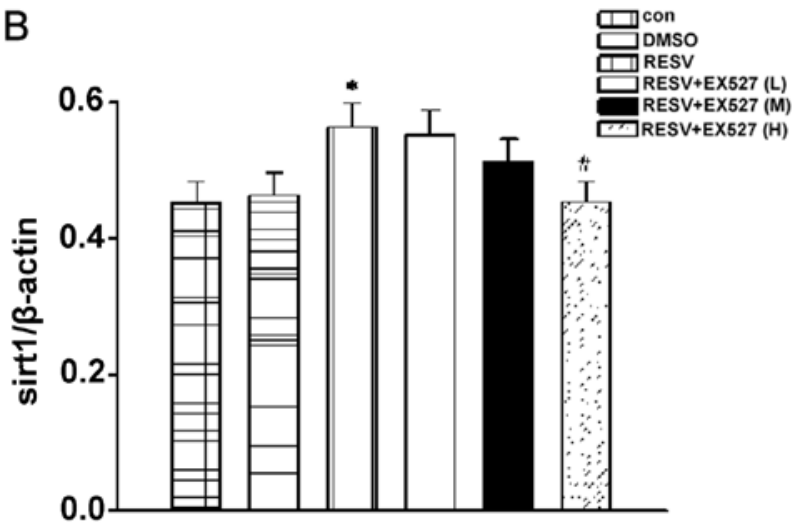

Figure 6. The EX527 function on SIRT1 protein expression in high glucose-exposed HepG2 cells as determined by western blot analysis. Cells were incubated in the presence of $\operatorname{EX527}(10,20$ and $40 \mu \mathrm{M}) 0.5 \mathrm{~h}$ before resveratrol $(100 \mu \mathrm{M})$ was added. (A) Representative western blots showing SIRT1 protein and $\beta$-actin in the same samples. (B) The abundance of SIRT1 and the downregulation of SIRT1 protein as a ratio to $\beta$-actin. The results represent the means \pm SD of three independent determinations ( ${ }^{*} \mathrm{P}<0.05$ compared with the control groups; ${ }^{*} \mathrm{P}<0.05$ compared with the resveratrol groups).
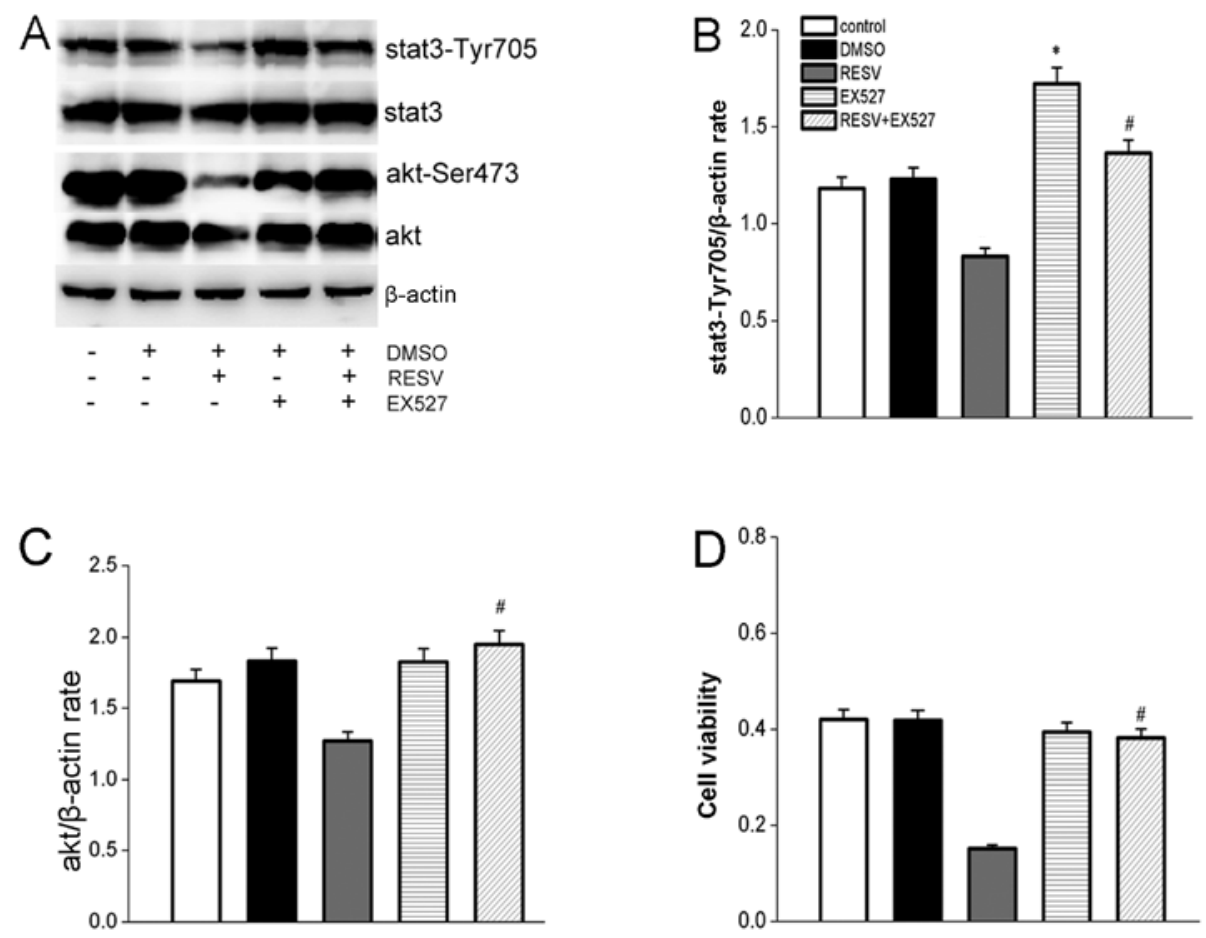

Figure 7. Effect of EX527 on the expression of P-STAT3, p-AKT and also on cell viability. Cells were incubated in the presence of EX527 (40 $\mu \mathrm{M}) 0.5 \mathrm{~h}$ before resveratrol $(100 \mu \mathrm{M})$ was added. (A) Representative western blots showing p-STAT3, STAT3, p-AKT, AKT and $\beta$-actin protein in the same samples. (B) The abundance of p-STAT3-Tyr705 and the abundance of p-STAT3-Tyr705 protein as a ratio to $\beta$-actin. (C) The abundance of p-AKT-Ser473 and the abundance of p-AKT-Ser473 protein as a ratio to $\beta$-actin. (D) Effect of resveratrol or EX527 on high glucose-induced cell viability in HepG2 cells. HepG2 cells were pretreated with EX527 for $0.5 \mathrm{~h}$, and the indicated concentrations of resveratrol were then added to the medium. After incubation for $12 \mathrm{~h}$ at $37^{\circ} \mathrm{C}$, cell viability was determined by the MTT reduction assay as described in Materials and methods. The results represent the means \pm SD of three independent determinations $\left({ }^{*} \mathrm{P}<0.05\right.$ compared with the resveratrol groups).

MMP-9, cyclin B1, cyclin D1 and VEGF, was investigated by reverse transcription-PCR. Analysis of HepG2 cells cultured in the presence of resveratrol $(100$ and $200 \mu \mathrm{M})$ for $24 \mathrm{~h}$ showed that the expression of MMP-9, cyclin B1 and VEGF was downregulated, although cyclin B1 expression was unaffected (Fig. 5A).

Resveratrol induces the expression of SIRT1 and suppresses the AKT signaling pathway. The expression of SIRT1 and
p-AKT by HepG2 cells under the influence of difference concentrations of resveratrol was investigated by western blot analysis. Resveratrol induced the overexpression of SIRT1 (Fig. 4A), while the expression of p-AKT was downregulated (Fig. 4A). Both effects of resveratrol were dose-dependent.

Role of EX527 in STAT3 and AKT signaling and cell viability. We analyzed the role of SIRT1 in STAT3 and AKT signaling 

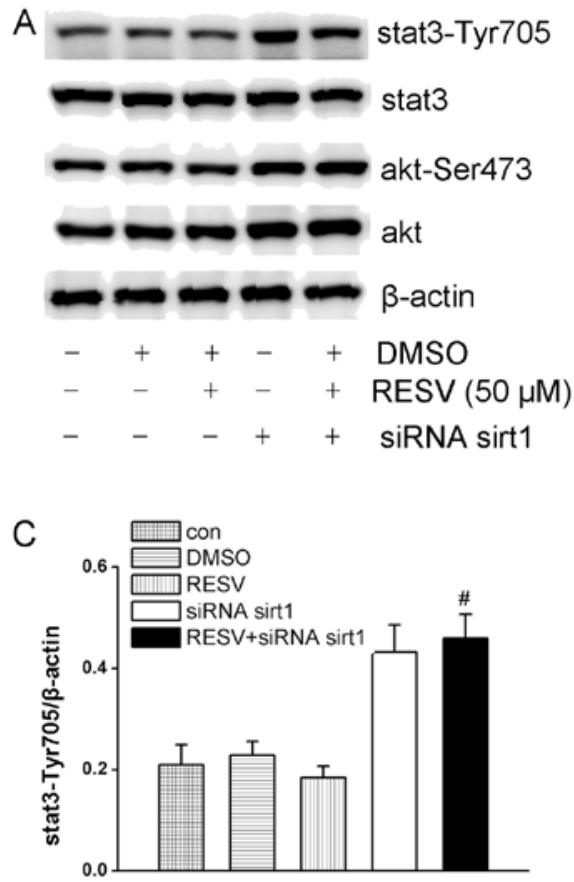

B

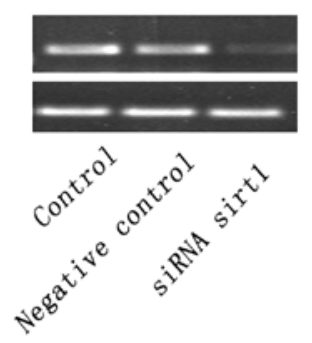

D

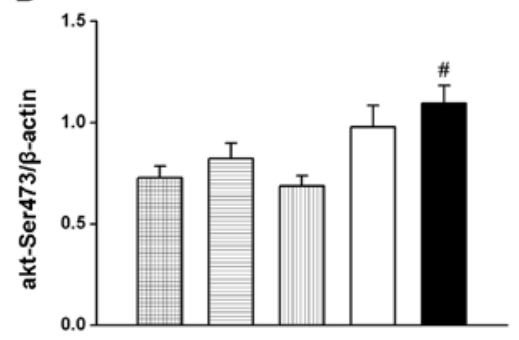

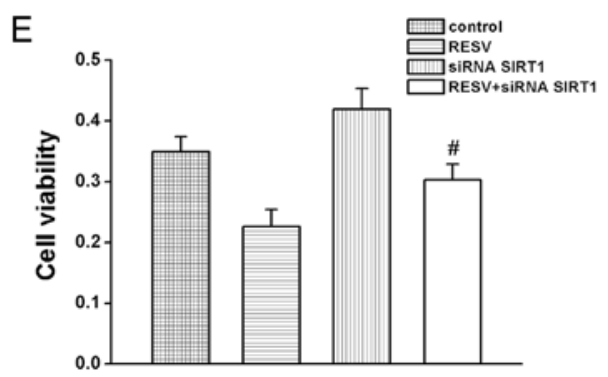

Figure 8. Effect of siRNA SIRT1 on the expression of P-STAT3, p-AKT and on cell viability. Cells were transfected with siRNA SIRT1 (10 $\mu \mathrm{M})$ for $48 \mathrm{~h}$ and then incubated in the presence of resveratrol $(50 \mu \mathrm{M})$. (A) Representative western blots showing p-STAT3, STAT3, p-AKT, AKT and $\beta$-actin protein in the same samples. (B) Representative ethidium bromide stained gels showing SIRT1 and $\beta$-actin in the same samples. (C) Abundance of p-STAT3-Tyr705 and the abundance of p-STAT3-Tyr705 protein as a ratio to $\beta$-actin. (D) The abundance of p-AKT-Ser473 and the abundance of p-AKT-Ser473 protein as a ratio to $\beta$-actin. (E) Effect of siRNA SIRT1 on resveratrol-induced cell viability in high glucose-exposed HepG2 cells. HepG2 cells were pretreated with siRNASIRT1 transfected for $48 \mathrm{~h}$, and the indicated concentrations of resveratrol were then added to the medium. After incubation for $12 \mathrm{~h}$ at $37^{\circ} \mathrm{C}$, cell viability was determined by the MTT reduction assay as described in Materials and methods. The results represent the means \pm SD of three independent determinations $\left({ }^{*} \mathrm{P}<0.05\right.$ compared with the resveratrol groups).

by using the SIRT1-specific inhibitor, EX527. SIRT1 expression in HepG2 cells was inhibited in a dose-dependent manner by pretreatment with EX527 (10, 20 and $40 \mu \mathrm{M})$ for $30 \mathrm{~min}$ (Fig. 6A). Furthermore, EX527 (40 $\mu \mathrm{M})$ suppressed the effects of resveratrol on STAT3 and AKT signaling; it weakened the effect of resveratrol function on p-STAT3 and p-AKT (Fig. 7A). EX527 also partially abrogated the suppression of resveratrol on HepG2 cell viability under a high glucose condition (Fig. 7D).

Effect of siRNA-SIRT1 on the STAT3 and AKT signaling pathways and cell viability. To investigate the role of SIRT1 in the effects of resveratrol on the STAT3 and AKT signaling pathways we used siRNA-SIRT1 $(10 \mu \mathrm{M})$ to downregulate the expression of SIRT1 at the level of transcription (Fig. 8A). We found that siRNA-SIRT1 weakened the suppressive function of resveratrol on the STAT3 and AKT signaling pathways. Furthermore, siRNA-SIRT1 exhibited an effect on the cell viability of HepG2 cells under the function of resveratrol.
These observations indicated that the effects of resveratrol on the STAT3 and AKT signaling pathways and cell viability in HepG2 cells under conditions of high glucose were partially SIRT1-dependent (Fig. 8A and E).

\section{Discussion}

The risk of hepatocellular carcinoma, which is the most common type of liver cancer, is increased in type 2 diabetic patients, although the reason for this is not clear (1). Resveratrol exerts important effect on cancer cell proliferation; therefore, elucidation of the mechanism of the effects of resveratrol in HepG2 cells under high glucose conditions may provide important insights into the reasons why the risk of hepatocellular carcinoma is greater in type 2 diabetic patients.

In the present study, we showed that a high glucose condition upregulated the expression of phospho-STAT3, and enhanced the viability of HepG2 cells (Fig. 1). Resveratrol suppressed the proliferation and invasive capacity of HepG2 
cells and also suppressed phospho-STAT3 and phospho-AKT expression. Furthermore, our data indicated that these effects were partly mediated through SIRT1. Thus, the present study indicated that resveratrol plays an important role in controlling the proliferation and invasion of high glucose-exposed HepG2 cells.

Resveratrol is a stilbenoid that is naturally produced by several plants. Although the capacity of resveratrol to prevent cancer development has been studied for many years (14), the mechanism underlying the efficacy of resveratrol, even at massive doses, remains to be fully elucidated (15). Recent research has shown that resveratrol suppresses proliferation and induces apoptosis in medulloblastoma cells by inhibition of STAT3 signaling and suppression of the expression of a range of cancer-associated genes (6). The effect of resveratrol on cancer has been demonstrated in other studies $(16,17)$ on malignant cells and medulloblastoma cells. These studies also indicate that the transcription factor STAT3 is regulated by resveratrol. STAT3, which plays an important role in cancer development, is a member of the STAT3 protein family. In response to cytokines and growth factors, STAT family members are phosphorylated by receptor-associated kinases and then form homodimers or heterodimers that translocate to the cell nucleus, where they act as transcription activators. In recent research, STAT3 was shown to have an oncogenic or tumor suppressor role depending on the mutational background of tumors. Evidence indicates that chronic STAT3 activation is a key event in gastric cancer induction and progression (18). Furthermore, increased STAT3 activity can upregulate the survival signal in cancer cells (19) and specific inhibition of STAT3 is a useful cancer treatment approach (20-22). In the present study, we found that resveratrol suppressed the expression of STAT3 and inhibited the proliferation of cancer cells although the function of resveratrol on the STAT3 signaling pathway in HepG2 cells and the effects of a high glucose concentration on the expression of STAT3 have not been investigated to date. In the present study, we found that resveratrol suppressed the STAT3 signaling pathway and inhibited the proliferation of high glucose-exposed HepG2 cells (Figs. 2, 4 and 5).

In the present study, we found that resveratrol induced the expression of SIRT1, which was the first sirtuin gene to be identified. Subsequently, other members of this highly conserved family have been found in nearly all organisms studied (23), SIRT2 is the only Class III histone deacetylase (HDAC) in budding yeast (24). The HDAC activity of SIRT2 results in tighter packaging of chromatin and a reduction in transcription at the targeted gene locus. Recently, the STAT3dependent effects of IL-22 in human keratinocytes were found to be counter-regulated by sirtuin SIRT1 through a direct inhibition of STAT3 acetylation (25). Furthermore, SIRT1 gene knock-out murine embryonic fibroblast (MEF) cells have been shown to induce expression of STAT3-tyr705 (26). These observations indicate that the STAT3 signaling pathway is regulated by SIRT1. The connection between acetylation and phosphorylation of STAT3 implies that STAT3 may have an important role in other cellular processes that involve SIRT1 (9). However, the relationship of SIRT1 and STAT3 in HepG2 cells has not been reported to date, and whether SIRT1 is involved in the effects of resveratrol on the suppression of
STAT3 has never been proven. In the present study, we demonstrated that the SIRT1 suppressor EX527 and siRNA-SIRT1 influenced the expression of SIRT1, indicating that SIRT1 is involved in the effects of resveratrol on the suppression of p-STAT3 and p-AKT as well as in the proliferation of HepG2 cells under a high glucose condition.

During our research, we found that resveratrol $(100 \mu \mathrm{M})$ suppressed the proliferation of HepG2 cells. In recent years, researchers have also found other factors that affect the proliferation of HepG2 cells, such as $\beta(2)$-AR agonists $\left(R, R^{\prime}\right)$-fenoterol (Fen) and (R,R')-4-methoxy-1-naphthylfenoterol (MNF), BBR and THP which inhibit growth $(25,27)$. There are many reports concerning the control of HepG2 cell proliferation, and the mechanism is complex. We hypothesize that the findings of the present study may help explain the increased risk of hepatocellular carcinoma in type 2 diabetic patients in whom glucose levels are much higher than normal and also provide insights into the mechanism by which resveratrol controls HepG2 cell proliferation. We also provided evidence clarifying the relationship between resveratrol, STAT3 and SIRT1 and demonstrated that the effect of resveratrol on the STAT3 signaling pathway is partly mediated via SIRT1 signaling.

In summary, resveratrol is a new cancer suppressor in high glucose-exposed HepG2 cells, and its mechanism may involve the STAT3 and AKT signaling pathways. Our observations may explain the increased risk of hepatocellular carcinoma in type 2 diabetic patients. However, the mechanism by which resveratrol controls HepG2 cell proliferation and the relationship of SIRT1 and STAT3 in this process warrants further research.

\section{Acknowledgements}

The present study was supported by grants from the National Natural Science Foundation of China (\#81070110 to M.W) and Shanghai Science and Tecnology Innovation Research Program (\#11410701900 to M.W). We would like to thank Jianping Tao and Yanwei Qing for their technical support.

\section{References}

1. El-Serag HB, Hampel $\mathrm{H}$ and Javadi F: The association between diabetes and hepatocellular carcinoma: a systematic review of epidemiologic evidence. Clin Gastroenterol Hepatol 4: 369-380, 2006.

2. Zheng M, Chen R, Zhong $\mathrm{H}$, et al: Side-effects of resveratrol in HepG2 cells: reduced pten and increased bcl-xl mRNA expression. Mol Med Rep 6: 1367-1370, 2012.

3. Bhardwaj A, Sethi G, Vadhan-Raj S, et al: Resveratrol inhibits proliferation, induces apoptosis, and overcomes chemoresistance through down-regulation of STAT3 and nuclear factor- $\kappa \mathrm{B}$ regulated antiapoptotic and cell survival gene products in human multiple myeloma cells. Blood 109: 2293-2302, 2007.

4. Capiralla H, Vingtdeux V, Venkatesh J, et al: Identification of potent small-molecule inhibitors of STAT3 with anti-inflammatory properties in RAW 264.7 macrophages. FEBS J 279: 3791-3799, 2012.

5. Haghikia A, Stapel B, Hoch M and Hilfiker-Kleiner D: STAT3 and cardiac remodeling. Heart Fail Rev 16: 35-47, 2011.

6. Wen $\mathrm{S}, \mathrm{Li} \mathrm{H}, \mathrm{Wu} \mathrm{ML}$, et al: Inhibition of NF- $\mathrm{B}$ signaling commits resveratrol-treated medulloblastoma cells to apoptosis without neuronal differentiation. J Neurooncol 104: 169-177, 2011.

7. Cho IR, Koh SS, Malilas W, et al: SIRT1 inhibits proliferation of pancreatic cancer cells expressing pancreatic adenocarcinoma up-regulated factor (PAUF), a novel oncogene, by suppression of $\beta$-catenin. Biochem Biophys Res Commun 423: 270-275, 2012. 
8. Kim YJ, Hwang SH, Lee SY, et al: miR-486-5p induces replicative senescence of human adipose tissue-derived mesenchymal stem cells and its expression is controlled by high glucose. Stem Cells Dev 21: 1749-1760, 2012.

9. Nie Y, Erion DM, Yuan Z, et al: STAT3 inhibition of gluconeogenesis is downregulated by SirT1. Nat Cell Biol 11: 492-500, 2009.

10. Li T, Wang W, Chen H, Li T and Ye L: Evaluation of antileukemia effect of resveratrol by modulating STAT3 signaling. Int Immunopharmacol 10: 18-25, 2010.

11. Pawlus MR, Wang L and Hu CJ: STAT3 and HIF1alpha cooperatively activate HIF1 target genes in MDA-MB-231 and RCC4 cells. Oncogene: Apr 22, 2013 (Epub ahead of print).

12. Li J, Cui G, Sun L, et al: STAT3 acetylation-induced promoter methylation is associated with downregulation of the ARHI tumor-suppressor gene in ovarian cancer. Oncol Rep 30: 165-170, 2013.

13. Absood A, Hu B, Bassily N and Colletti L: VIP inhibits human HepG2 cell proliferation in vitro. Regul Pept 146: 285-292, 2008

14. Baur JA and Sinclair DA: Therapeutic potential of resveratrol: the in vivo evidence. Nat Rev Drug Discov 5: 493-506, 2006.

15. Athar M, Back JH, Tang X, et al: Resveratrol: a review of preclinical studies for human cancer prevention. Toxicol Appl Pharmacol 224: 274-283, 2007.

16. Kotha A, Sekharam M, Cilenti L, et al: Resveratrol inhibits Src and Stat 3 signaling and induces the apoptosis of malignant cells containing activated Stat 3 protein. Mol Cancer Ther 5: 621-629, 2006.

17. Yu LJ, Wu ML, Li H, et al: Inhibition of STAT3 expression and signaling in resveratrol-differentiated medulloblastoma cells. Neoplasia 10: 736-744, 2008.

18. Giraud AS, Menheniott TR and Judd LM: Targeting STAT3 in gastric cancer. Expert Opin Ther Targets 16: 889-901, 2012.
19. Deng J, Liu Y, Lee H, et al: S1PR1-STAT3 signaling is crucial for myeloid cell colonization at future metastatic sites. Cancer Cell 21: 642-654, 2012.

20. Tkach M, Coria L, Rosemblit C, et al: Targeting Stat 3 induces senescence in tumor cells and elicits prophylactic and therapeutic immune responses against breast cancer growth mediated by NK cells and CD4 ${ }^{+}$T cells. J Immunol 189: 1162-1172, 2012.

21. Sen M, Joyce S, Panahandeh M, et al: Targeting Stat3 abrogates EGFR inhibitor resistance in cancer. Clin Cancer Res 18: 4986-4996, 2012.

22. Yang CL, Liu YY, Ma YG, et al: Curcumin blocks small cell lung cancer cell migration, invasion, angiogenesis, cell cycle and neoplasia through Janus kinase-STAT3 signalling pathway. PLoS One 7: e37960, 2012.

23. Frye RA: Phylogenetic classification of prokaryotic and eukaryotic Sir2-like proteins. Biochem Biophys Res Commun 273: 793-798, 2000.

24. Chang KT and Min KT: Regulation of lifespan by histone deacetylase. Ageing Res Rev 1: 313-326, 2002.

25. Sestito R, Madonna S, Scarponi C, et al: STAT3-dependent effects of IL-22 in human keratinocytes are counterregulated by sirtuin 1 through a direct inhibition of STAT3 acetylation. FASEB J 25: 916-927, 2011.

26. Bernier M, Paul RK, Martin-Montalvo A, et al: Negative regulation of STAT3 protein-mediated cellular respiration by SIRT1 protein. J Biol Chem 286: 19270-19279, 2011.

27. Chen X, Cao Y, Lv D, Zhu Z, Zhang J and Chai Y: Comprehensive two-dimensional HepG2/cell membrane chromatography/ monolithic column/time-of-flight mass spectrometry system for screening anti-tumor components from herbal medicines. J Chromatogr A 1242: 67-74, 2012. 